Совершенствование рецептуры резины для массивных шин путем добавок нетрадиционных наполнителей

\begin{tabular}{llll}
\hline \hline Инна А. Литвинова & 1 & innalitv@mail.ru & (D) 0000-0001-5359-1631 \\
Игорь В. Веселов & 2 & iv-veselov@mail.ru & \\
Юрий А. Гамлицкий & ${ }^{2}$ & gamlit48@ mail.ru & (D) 0000-0001-8925-2439 \\
\hline
\end{tabular}

${ }^{1}$ МИРЭА - Российский технологический университет, пр-т Вернадского, д. 78, г. Москва, 119454, Россия

2 ООО НПКЦ ВЕСКОМ, ул. Буракова, 27, г. Москва, 105118, Россия

Аннотация. Исследовали влияние новых наполнителей на свойства резин для массивных шин. В качестве новых наполнителей использовали модифицированные фуллеренами и немодифицированные железооксидные наполнители (ЖОН). Усталостные характеристики определяли двумя методами: многократное растяжение на приборе УР-500 в соответствии с ГОСТ 261-79; знакопеременный изгиб с вращением на приборе СЗПИ в соответствии с ГОСТ 10952-75. Основные задачи построения усталостных кривых - оценка соответствия результатов прочностных испытаний (растяжение с постоянной скоростью до разрыва) усталостным по показателям усталостной долговечности, усталостной прочности, усталостной плотности энергии разрушения. Кроме того, стояла задача проверки возможности нанесения на одну кривую Веллера точек, полученных на разных приборах, - УР 500 и ЗПИ. Ранжирование резин в статике не совпадает друг с другом. Так как усталостные условия нагружения ближе к условиям реальной эксплуатации, чем статические, то для прогнозирования поведения РТИ в эксплуатации следует ориентироваться на результаты усталостных испытаний. Совмещение результатов усталостных испытаний при многократном растяжении и при знакопеременном изгибе с вращением показало, что эта процедура правомерна, но только в тех случаях, когда температура саморазогрева на приборе ЗПИ не превышает некоторого критического значения, когда начинается термодеструкция. Результаты усталостных испытаний показали, что использование исследованных наполнителей при небольших степенях наполнения (до 30 массовых частей) может быть оправдано. В качестве примера можно привести Ferrocolor фракции 0-20 Мкм с массовой долей 5 м.ч. В этом случае усталостные свойства практически не ухудшаются по сравнению с промышленной резиной для массивных шин, в которую не добавлены новые наполнители.

Ключевые слова: резина, активный наполнитель, усиление, прочность, усталостные характеристики

\title{
Improvement of rubber recipe for massive tires by addition of non- traditional fillers
}

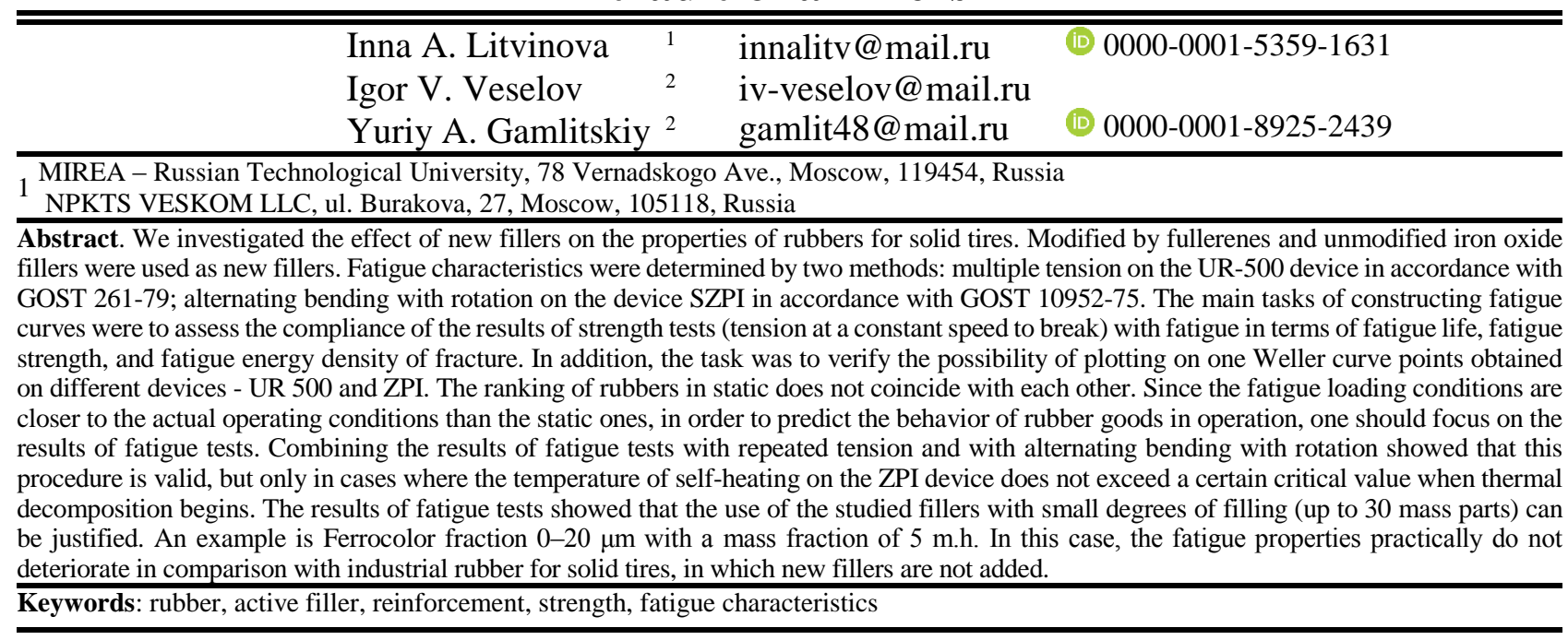

\section{Введение}

Результаты статических испытаний резин не позволяют предвидеть их усталостные свойства. Накопленный большой экспериментальный материал и прогресс в понимании закономерностей усталостной выносливости дают основание поставить вопрос о преимущественном использовании показателей усталостной выносливости для

Для цитирования

Литвинова И.А., Веселов И.В., Гамлицкий Ю.А. Совершенствование рецептуры резины для массивных шин путем добавок нетрадиционных наполнителей // Вестник ВГУИТ. 2019. Т. 81. № 4. С. 196-204. doi:10.20914/2310-1202-2019-4-196-204

(C) 2019, Литвинова И.А. и др./ Litvinova I.A. et al. оценки качества резин, работающих при циклическом нагружении, как наиболее точной и достоверной методологии по сравнению с практикой применения для этой цели показателей статических испытаний [1].

Основные требования к работоспособности массивных шин: 1) низкие гистерезисные потери, 2) высокий уровень усталостных свойств, 3) хорошая адгезия резины к металлу диска.

For citation

Litvinova I.A., Veselov I.V., Gamlitskiy Yu.A. Improvement of rubber recipe for massive tires by addition of non-traditional fillers. Vestnik VGUIT [Proceedings of VSUET]. 2019. vol. 81. no. 4. pp. 196-204. (in Russian). doi:10.20914/2310-1202-2019-4-196-204

This is an open access article distributed under the terms of the Creative Commons Attribution 4.0 International License 
В данной работе ставится задача оценки усталостных свойств модифицированных резин для массивных шин. Усталостные испытания проводили на машинах УР-500 (многократное растяжение) и ЗПИ (знакопеременный изгиб с вращением). Кривые Веллера строили в трёх режимах: заданных деформаций, заданных напряжений, заданных плотностей энергии деформации.

Также поставлена задача построить кривые Веллера по результатам усталостных испытаний на двух усталостных машинах - УР-500 и ЗПИ [3] на одном графике. До настоящего времени считалось, что результаты испытаний по разным методикам несовместимы. В работе поставлена задача проверить это утверждение.

В качестве объектов исследования выбраны промышленная резина для массивных шин и те же резины с добавлением разных железооксидных наполнителей (ЖОН): FerroFlex, FerroColor [7].

\section{Материалы и методы}

В качестве исследуемых наполнителей были выбраны ЖОН (модифицированные фуллеренами и немодифицированные) с разным размером частиц.

FerroFlex (FF) - комбинированный активный наполнитель армирующего свойства. Представляет собой комплексную систему, состоящую из высокодисперсного железоокисного материала, модифицированного фуллеренами, органическими кислотами, волокнистыми минеральными наполнителями,

активным углеродом. Влияет на следующие характеристики резин: напряжение при растяжении, прочность при разрыве, твёрдость по Шору, эластичность по отскоку, сопротивление истиранию, относительное удлинение при разрыве, стойкость к окислению. Объёмные характеристики:

- $\quad$ кажущаяся (насыпная) плотность $p=1,1$ г/ $\mathrm{cm}^{3}$;

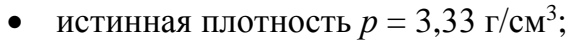

- основная фракция - 10 мкм.

Наполнитель FerroColor (FC) представляет собой высокодисперсный железоокисный материал, аналогичный FerroFlex, отличающийся от FerroFlex только тем, что не модифицирован фуллеренами [4].

Упруго-прочностные свойства резин определяли на машине УТС-5 в соответствии с ГОСТ 270, ГОСТ 262 и ГОСТ 252.

\section{Результаты}

Испытания проводили для резины массивных шин (таблица 1), в которую добавляли железооксидные наполнители FF в количестве $5,10,20,40$ м. ч. и FC разных фракций: $(0-20$ мкм): 5,10, 20, 24 м. ч.; (0-100 мкм): 5, 10, 20, $30,30,5$ м. ч.

Результаты физико-механических испытаний резин с наполнителем FF приведены в таблице 2. На рисунках 1-3 представлены кривые растяжения. Отметим, что продолжение кривых растяжения после точки разрыва не имеет смысловой нагрузки. В таком виде испытательная машина выдаёт результаты.

Таблица 1 .

Рецептура резин для изготовления массивных шин

Table 1.

Recipe of rubber for the manufacture of solid tires

\begin{tabular}{|c|c|}
\hline \multicolumn{2}{|c|}{ Основные ингредиенты для шин клеевого крепления | Main ingredients for tires Adhesive fastening } \\
\hline Каучук СКМС-10К | Rubber SKMS-10К & 70,00 \\
\hline Каучук СКМС-30АРКМ-15 | Rubber SKMS-30ARKM-15 & 30,00 \\
\hline Углерод технический ПМ-100 или ПМ-105| Technical carbon PM-100 or PM-105 & 15,00 \\
\hline Углерод технический ПМ-50| Technical carbon PM-50 & 50,00 \\
\hline
\end{tabular}

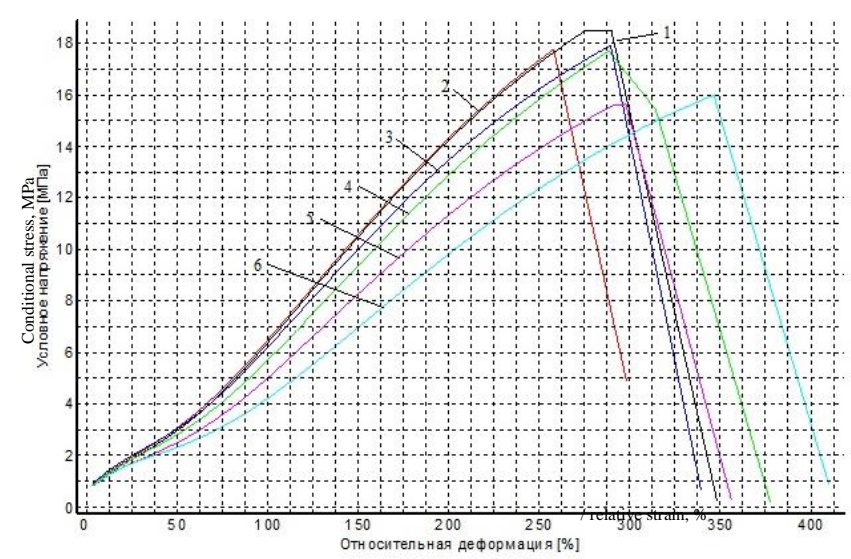

Рисунок 1. Кривые растяжения для резин с FerroFlex: 1 - эталон; 2 - 5 м.ч. FF; 3 - 10 м.ч. FF; 4 - 20 м.ч. FF; 5 - 30 м.ч. FF; 6 - 40 м.ч. FF

Figure 1. Tensile Curves for Rubber with FerroFlex: 1 - reference; $2-5$ m.h. FF; $3-10$ m.h. FF; $4-20$ m.h. FF; $5-30$ m.h. FF; $6-40$ m.h. FF 


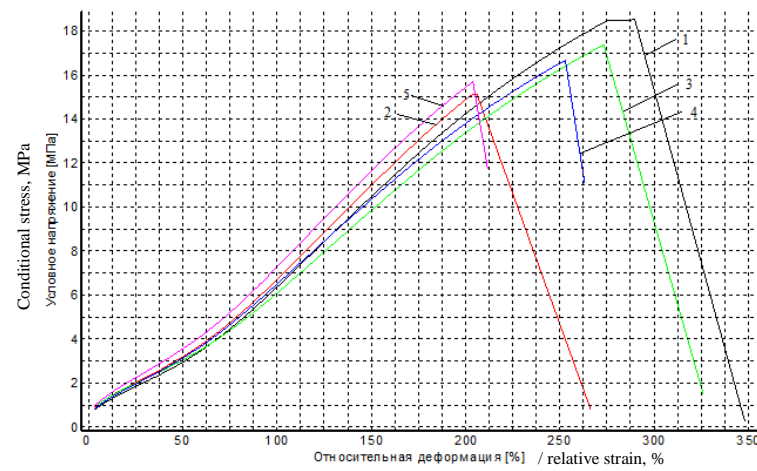

Рисунок 2. Кривые растяжения серии испытаний резины с FerroColor (0-20 мкм): 1 - эталон; 2 - 5 м.ч. FC (0-20 мкм); 3 - 10 м.ч. FC (0-20 мкм); 4 - 20 м.ч. FC (0-20 мкм); 5 - 24 м.ч. FC (0-20 мкм)

Figure 2. Tensile curves of a series of tests of rubber with FC (0-20 microns): 1 - standard; $2-5$ m.h FC (020 microns); $3-10$ m.h. FC (0-20 microns); $4-20$ m.h. FC (0-20 microns); 5 - 24 m.h. FC (0-20 microns)

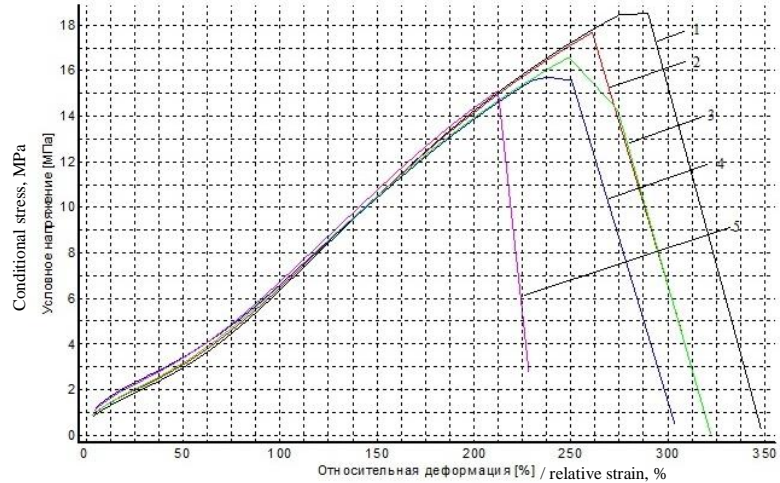

Рисунок 3. Кривые растяжения для резин с FerroColor (0-100) мкм: 1 - эталон; 2 - 10 м.ч. FC (0$100)$ мкм; 3 - 20 м.ч. FC (0-100) мкм; $4-30$ м.ч. FC (0-100) мкм; 5 - 30,5 м.ч. FC (0-100) мкм

Figure 3. Tension curves for rubbers with FerroColor (0-100) microns: 1 - standard; 2-10 m.h FC (0-100) microns; 3 - 20 m.h. FC (0-100) microns; $4-30$ m.h. FC (0-100) microns; 5 - 30.5 m.h FC (0-100) microns

Таблица 2 .

Результаты физико-механических испытаний резин для массивных шин

Table 2.

Results of physical and mechanical testing of rubber for massive tires

\begin{tabular}{|c|c|c|c|c|c|c|c|c|c|c|c|c|c|}
\hline \multirow[b]{2}{*}{$\begin{array}{l}\text { Показатель } \\
\text { Indicator }\end{array}$} & \multicolumn{13}{|c|}{ Шифр резины\ Rubber code } \\
\hline & 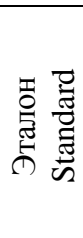 & 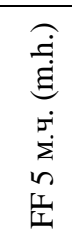 & 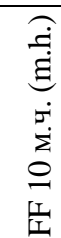 & 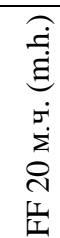 & 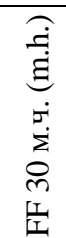 & 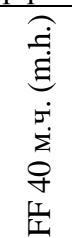 & 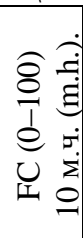 & 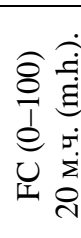 & 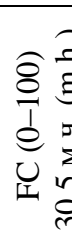 & 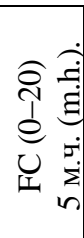 & 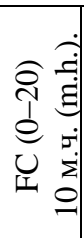 & 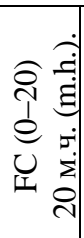 & 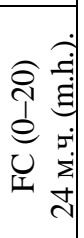 \\
\hline $\begin{array}{c}\text { Относительный гистерезис, \% } \\
\text { Relative hysteresis, \% }\end{array}$ & 11,2 & 9,9 & 9,7 & 8,3 & 12,3 & 10,4 & 9,2 & 9,5 & 9,6 & 9,4 & 8,9 & 9,9 & 10,4 \\
\hline $\begin{array}{c}\text { Прочность при разрыве, Мпа } \\
\text { Tensile strength, MPa }\end{array}$ & 17,7 & 17,8 & 17,3 & 17,5 & 16,4 & 15,7 & 16,5 & 16,6 & 14,9 & 15,3 & 16,3 & 16,2 & 15,8 \\
\hline $\begin{array}{c}\text { Относительное удлинение при } \\
\text { разрыве, } \% \\
\text { Elongation at break, \% }\end{array}$ & 265 & 262 & 276 & 278 & 312 & 337 & 243 & 245 & 212 & 208 & 253 & 241 & 215 \\
\hline $\begin{array}{c}\text { Сопротивление раздиру Н/мм } \\
\text { Tear resistance N/mm }\end{array}$ & 54 & 51 & 45 & 45 & 57 & 54 & 49 & 52 & 43 & 53 & 56 & 48 & 39 \\
\hline
\end{tabular}

В таблице 3 приведены данные по усталостной выносливости в режиме заданных деформаций для эталонной резины (без железооксидного наполнителя) и с добавлением наполнителей FerroFlex и FerroColor.
На рисунке 4 представлены кривые Веллера в режиме заданных деформаций для резин с различным содержанием наполнителя FerroFlex; для резин FC (0-20); для резин с FC (0-100).

Таблица 3.

Данные по усталостной выносливости в режиме заданных деформаций для эталонной резины

Data on fatigue endurance for Preset Deformations for Reference Rubber

Table 3.

\begin{tabular}{|c|c|c|c|c|c|}
\hline $\begin{array}{c}\text { Шифр резины } \\
\text { Rubber code }\end{array}$ & $\begin{array}{c}\text { N, количество } \\
\text { циклов } \\
\text { number of cycles }\end{array}$ & $\operatorname{lgN}$ & $\begin{array}{c}\text { Деформация, } \varepsilon, \% \\
\text { Deformation }\end{array}$ & $\lg \varepsilon$ & $\begin{array}{c}\text { T, С (через 10 мин после начала } \\
\text { испытания) } \\
\text { (10 minutes after the start of the test) }\end{array}$ \\
\hline 1 & 2 & 3 & 4 & 5 & 6 \\
\hline \multirow{3}{*}{ Эталон | Standard } & 5459280 & 6,73 & 21,62 & 1,33 & 84,3 \\
\cline { 2 - 6 } & 1873672 & 6,27 & 24,65 & 1,39 & 89,8 \\
\cline { 2 - 6 } & 89976 & 4,95 & 30,72 & 1,48 & 110,6 \\
\hline \multirow{2}{*}{ FF 5 м.ч. (m.h.) } & 1268680 & 6,10 & 21,62 & 1,33 & 84,5 \\
\cline { 2 - 6 } & 984400 & 5,99 & 24,65 & 1,39 & 89,8 \\
\hline
\end{tabular}


Продолжение табл. 3 Continuation of table 3

\begin{tabular}{|c|c|c|c|c|c|}
\hline 1 & 2 & 3 & 4 & 5 & 6 \\
\hline \multirow{3}{*}{ FF 10 м.ч. (m.h.) } & 976120 & 5,98 & 21,62 & 1,33 & 92,6 \\
\hline & 150627 & 5,17 & 24,65 & 1,39 & 98,0 \\
\hline & 67344 & 4,82 & 30,72 & 1,48 & 108,8 \\
\hline \multirow{3}{*}{ FF 20 м.ч. (m.h.) } & 679420 & 5,83 & 21,62 & 1,33 & 83,7 \\
\hline & 431112 & 5,63 & 24,65 & 1,39 & 94,2 \\
\hline & 11040 & 4,04 & 30,72 & 1,48 & 130,0 \\
\hline \multirow{4}{*}{ FF 30 м.ч. (m.h.) } & 1759500 & 6,24 & 18,58 & 1,26 & 84,3 \\
\hline & 139840 & 5,14 & 21,62 & 1,33 & 98,8 \\
\hline & 300840 & 5,47 & 24,65 & 1,39 & 99,2 \\
\hline & 10448 & 4.01 & 30,72 & 1,48 & 130,0 \\
\hline \multirow{2}{*}{ FF 40 м.ч. (m.h.) } & 441048 & 5,64 & 21,62 & 1,33 & 90,8 \\
\hline & 219420 & 5,34 & 24,65 & 1,39 & 101,0 \\
\hline \multirow{3}{*}{ FC (0-20) 5 м.ч. (m.h.). } & 2470200 & 6,39 & 21,62 & 1,33 & 84,0 \\
\hline & 1765296 & 6,24 & 24,65 & 1,39 & 96,7 \\
\hline & 94944 & 4,97 & 30,72 & 1,48 & 96,7 \\
\hline \multirow{2}{*}{ FC (0-20) 10 м.ч. (m.h.). } & 660100 & 5,82 & 21,62 & 1,33 & 85,6 \\
\hline & 339420 & 5,53 & 24,65 & 1,39 & 97,2 \\
\hline \multirow{2}{*}{ FC (0-20) 20 м.ч. (m.h.). } & 756240 & 5,88 & 21,62 & 1,33 & 88,7 \\
\hline & 428888 & 5,63 & 24,65 & 1,39 & 89,2 \\
\hline \multirow{2}{*}{ FC (0-20) 24 м.ч. (m.h.). } & 92920 & 4,97 & 21,62 & 1,33 & 87.6 \\
\hline & 464784 & 5,66 & 24,65 & 1,39 & 107,8 \\
\hline \multirow{2}{*}{ FC (0-100) 10 м.ч. (m.h.). } & 903440 & 5,95 & 21,62 & 1,33 & 87,7 \\
\hline & 231560 & 5,36 & 24,65 & 1,39 & 97,3 \\
\hline \multirow{2}{*}{ FC (0-100) 20 м.ч. (m.h.). } & 218960 & 5,34 & 21,62 & 1,33 & 86,0 \\
\hline & 218933 & 5,34 & 24,65 & 1,39 & 95,1 \\
\hline \multirow{2}{*}{ FC (0-100) 30 м.ч. (m.h.). } & 105373 & 5,02 & 21,62 & 1,33 & 104,7 \\
\hline & 100740 & 5,00 & 24,65 & 1,39 & 107,5 \\
\hline \multirow{2}{*}{$\begin{array}{c}\mathrm{FC}(0-100) \\
30.5 \text { м.ч. (m.h.). }\end{array}$} & 148580 & 5,17 & 21,62 & 1,33 & 101,6 \\
\hline & 137386 & 5,13 & 24,65 & 1,39 & 101,8 \\
\hline
\end{tabular}

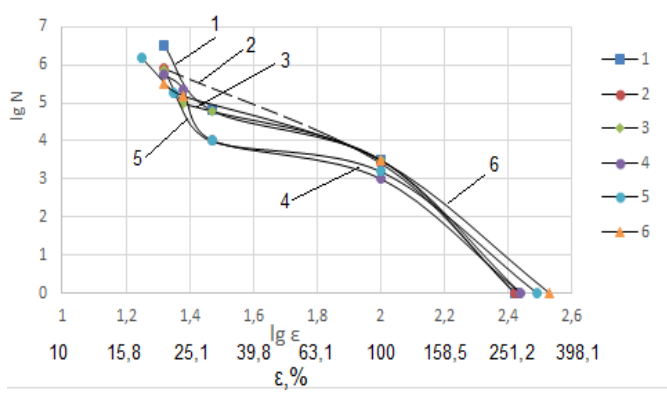

4 - FerroFlex 20 м.; ч 5 - FerroFlex 30 м. ч.; 6 - FerroFlex 40 м. ч.

1 - Standard; 2 - FerroFlex 5 m.h.; 3 - FerroFlex 10 m.h.; 4 - FerroFlex 20 m.h.; 5 - FerroFlex 30 m.h.; 6 FerroFlex 40 m.h.

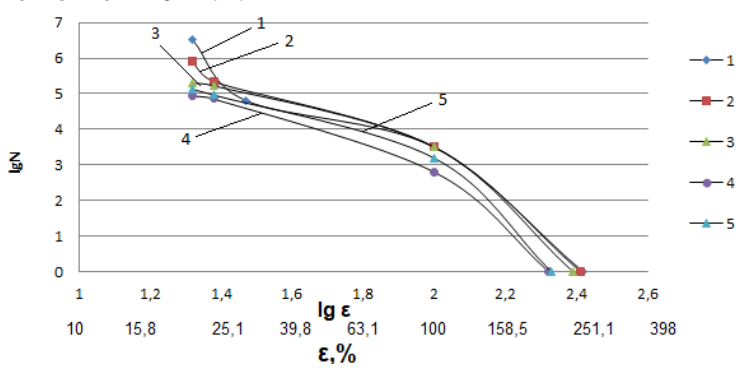

1 - Эталон; 2 - FC (0-100) 10 м.ч; 3 - FC (0-100) 20 м. ч 4) FC (0-100) 30 м. ч; 5 - FC (0-100) 30, 5 м. ч;

1 - Standard; 2 - FC (0-100) 10 m.h; 3 - FC (0-100) 20 m.h;

4 - FC (0-100) 30 m.h; 5 - FC (0-100) 30, 5m. h

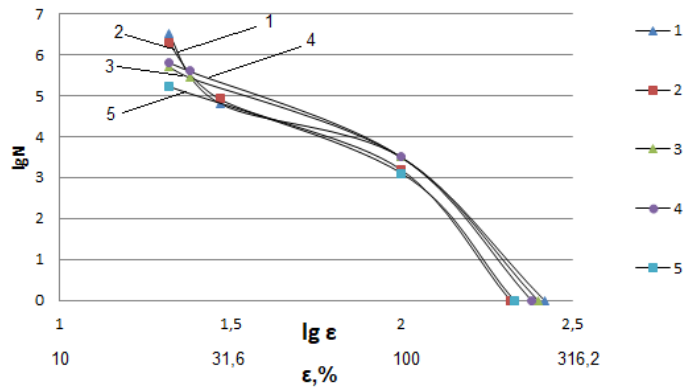

(b)

1 - Эталон; 2 - FC (0-20) 5 м.ч; 3 - FC (0-20) 10 м.ч.; 4 - FC (0-20) 20 м.ч; 5- FC (0-20) 24 м.ч

1 - Standard; 2 - FC (0-20) 5 m.h; 3 - FC (0-20) 10 m.h.;

4 - FC (0-20) 20 m.h; 5 - FC (0-20) 24 m.h

(c)

$-4$

Рисунок 4. Кривые Веллера в режиме заданных деформаций: $a$ - для резин с наполнителем FerroFlex; $b$ - для резин FC (0-20); $c$ - для резин с FC (0-100)

Figure 4. Weller curves in the set deformation mode: $a$ - for rubber with FerroFlex filler; $b$ - for FC rubber (0-20); $c$ - for FC rubber $(0-100)$ 
Далее пересчитали данные для кривых Веллера из режима заданных деформаций в режим заданных напряжений. Это было сделано с помощью кривых напряжения-деформации для данных резин, рисунки 1-3.

На рисунке 5 показаны кривые Веллера в режиме заданных напряжений для резин с FF; для резин с FC (0-20); для резин $\mathrm{FC}(0-100)$.

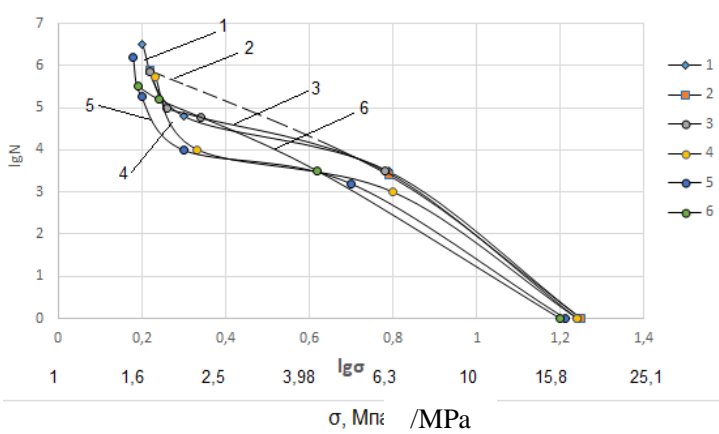

(a)

1 - Эталон; 2 - FF 5; 3 - FF 10; 4 - FF 20; 5 - FF 30; 6 - FF 40

1 - Standart; 2 - FF 5; 3 - FF 10; 4 - FF 20; 5 - FF 30; 6 - FF 40

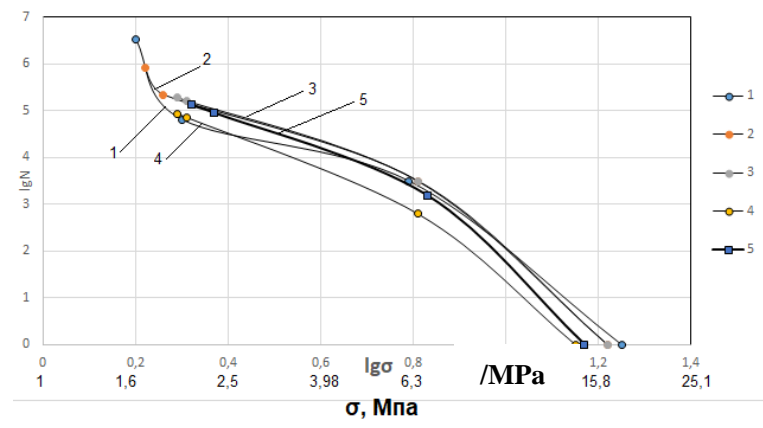

(c)
На рисунке 6 показаны кривые Веллера в режиме заданных плотностей энергии деформации для резин с FF; для резин с FC (0-20); для резин FC (0-100).

На рисунке 7 показаны гистограммы ходимости для резин с FF; для резин с FC (0-20); для резин $\mathrm{FC}(0-100)$.

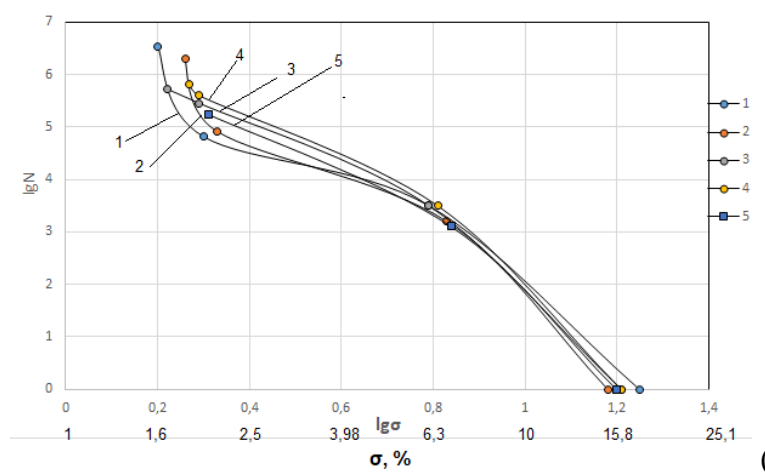

(b)

1 - Эталон; 2 - FC (0-20) 5 м.ч; 3 - FC (0-20) 10 м.ч.; 4 - FC (0-20) 20 м.ч; 5 - FC (0-20) 24 м.ч

1 - Standard; 2 - FC (0-20) 5 m.h; 3 - FC (0-20) 10 m.h; 4 - FC (0-20) 20 m.h; 5 - FC (0-20) 24 m.h

Рисунок 5. Кривые Веллера в режиме заданных напряжений: $a$ - для резин с FF; $b$ - для резин FC (0-20); $c$ - для резин с FC (0-100)

Figure 5. Weller curves in the set deformation mode: $a$ - for rubber with FF; $b$ - for FC rubber (0-20); $c$ - for FC rubber (0-100)

1 - Эталон; 2 - FC (0-100) 10 м.ч; 3 - FC (0-100) 20 м. ч; 4 - FC (0-100) 30 м. ч; 5 - FC (0-100) 30, 5 м. ч

1 - Standard; 2 - FC (0-100) 10 m.h; 3 - FC (0-100) 20 m.h; 4

- FC (0-100) 30 m.h; 5 - FC (0-100) 30.5 m. h

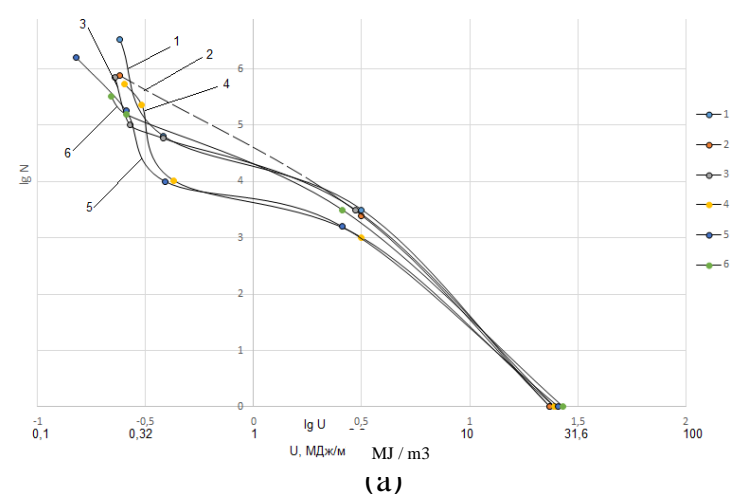

1 - Эталон; 2 - FF 5; 3 - FF 10; 4 - FF 20; 5 - FF 30; 6 - FF 40

1 - Standart; 2 - FF 5; 3 - FF 10; 4 - FF 20; 5 - FF 30; 6 - FF 40

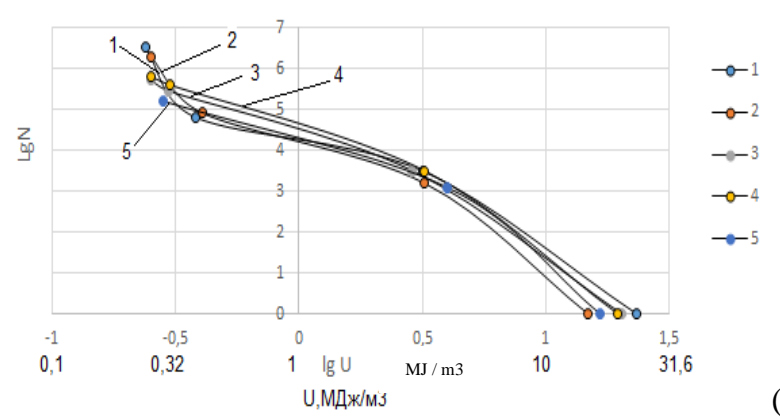

(b)

1 - Эталон; 2 - FC (0-20) 5 м.ч; 3 - FC (0-20) 10 м.ч.; 4 - FC (0-20) 20 м.ч; 5 - FC (0-20) 24 м.ч

1 - Standard; 2 - FC (0-20) 5 m.h; 3 - FC (0-20) 10 m.h; 4 - FC (0-20) 20 m.h; 5 - FC (0-20) 24 m.h 
Кривые Веллера в режиме заданныхплотностей энергии деформации для резин ЖОН 0-100

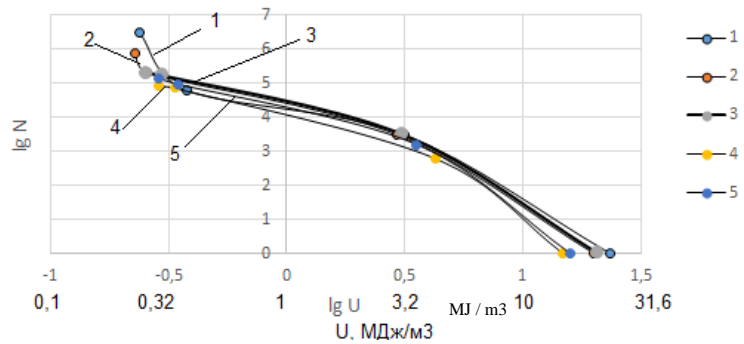

(c)

Рисунок 6. Кривые Веллера в режиме заданных плотностей энергии деформации: а - для резин с FF; $\mathrm{b}$ - для резин FC (0-20); c - для резин с FC (0-100)

Figure 6. Weller curves in the mode of preset deformation energy densities: $\mathrm{a}$ - for rubber with FF; $\mathrm{b}$ - for FC rubber (0-20); c - for FC rubber (0-100)

1 - Эталон; 2 - FC (0-100) 10 м.ч; 3 - FC (0-100) 20 м. ч; $4-\mathrm{FC}(0-100) 30$ м. ч; $5-\mathrm{FC}(0-100) 30,5$ м. ч

1 -Standard; $2-\mathrm{FC}(0-100) 10$ m.h; 3-FC (0-100) 20 m.h; $4-\mathrm{FC}(0-100) 30$ m.h; 5 - FC (0-100) $30.5 \mathrm{~m} . \mathrm{h}$

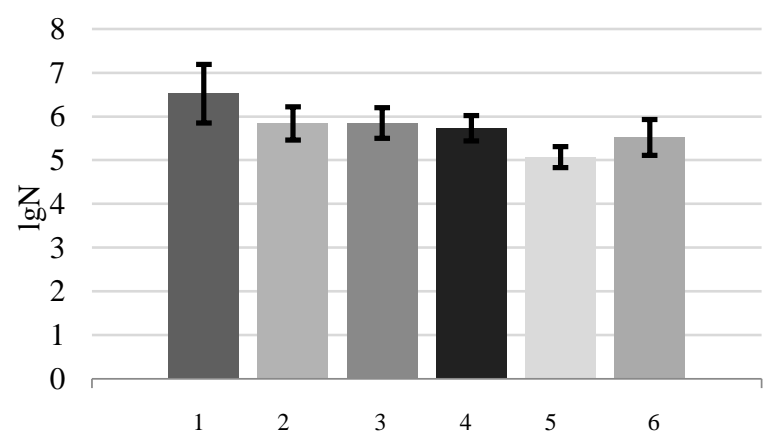

(a)

1 - Эталон; 2 - FF 5; 3 - FF 10; 4 - FF 20; 5 - FF 30; 6 - FF 40

1 - Standart; 2 - FF 5; 3 - FF 10; 4 - FF 20; 5 - FF 30; 6 - FF 40

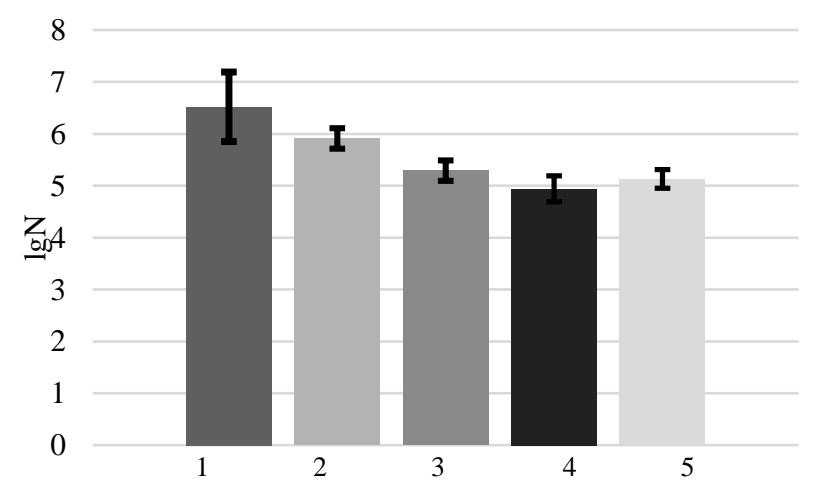

(c)

1 - Эталон; 2 - FC (0-100) 10 м.ч; 3 - FC (0-100) 20 м. ч; 4 - FC (0-100) 30 м. ч; 5 - FC (0-100) 30, 5 м. ч

1 - Standard; 2 - FC (0-100) 10 m.h; 3 - FC (0-100) 20 m.h; 4 - FC (0-100) 30 m.h; 5 - FC (0-100) 30.5 m. h

\section{Обсуждении}

Прочность эталонной резины составляет 17,7 МПа, относительное удлинение - 265\%. При введении железооксидного наполнителя Ferroflex в резины в количестве до 20 м. ч. прочность резин практически не ухудшается,

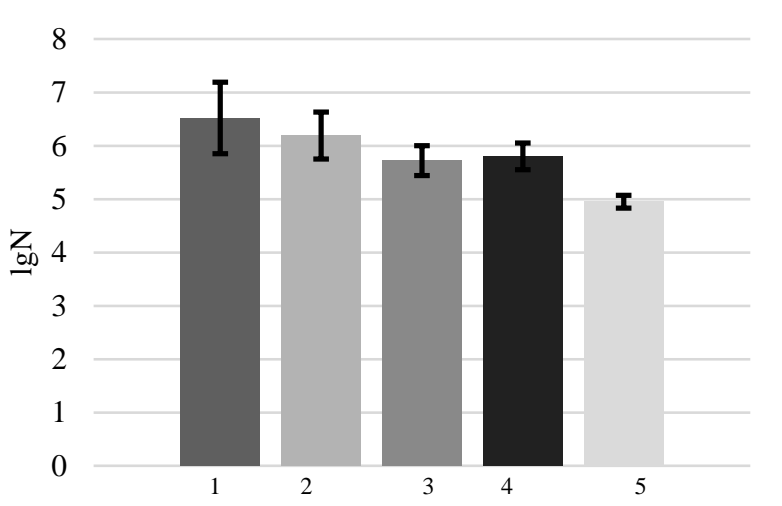

(b)

1 - Эталон; 2 - FC (0-20) 5 м.ч; 3 - FC (0-20) 10 м.ч.; 4 - FC (0-20) 20 м.ч; 5 - FC (0-20) 24 м.ч

1 - Standard; 2 - FC (0-20) 5 m.h; 3 - FC (0-20) 10 m.h; 4 - FC (0-20) 20 m.h; 5 - FC (0-20) 24 m.h

Рисунок 7. Гистограмма ходимости резин при деформации $21,62 \%$ : a - для резин с FF; $\mathrm{b}$ - для резин FC (0-20); с - для резин с FC (0-100)

Figure 7. Histogram of rubber mileage at a strain of $21,62 \%$ : a - for rubber with FF; b - for FC rubber $(0-20)$; $\mathrm{c}-$ for FC rubber (0-100)

также происходит небольшое увеличение относительного удлинения. По рисунку 1 видно, что с увеличением дозы наполнителя FerroFlex в резинах их модуль снижается. Это может быть связано с тем, что поверхность наполнителя модифицирована фуллеренами, он начинает 
взаимодействовать с серой, снижая тем самым густоту вулканизационной сетки, что приводит к снижению модуля резины [2].

При введении в резины наполнителя немодифицированного фракции 0-20 мкм эти показатели незначительно снижаются. Также по кривым растяжения (рисунок 2) видно, что для некоторых точек наполнения жёсткость увеличивается (24 м. ч.), а для некоторых уменьшается (10 масс. ч.). Здесь присутствует два фактора: в первом случае преобладает гидродинамический эффект, во втором начинается взаимодействие наполнителя с серой, вследствие чего уменьшается густота вулканизационной сетки и происходит уменьшение модуля резин [1]. Для более точной интерпретации этих результатов на основе теории усиления требуются дополнительные исследования.

В таблице 2 представлены результаты физико-механических испытаний резин с FC фракции 0-100 мкм.

По кривым растяжения (рисунок 3) видно, что с увеличением количества наполнителя прочность постепенно снижается. Жёсткость резин практически не меняется, что можно объяснить взаимной компенсацией влияния на модули гидродинамического эффекта и адсорбцией вулканизующей группы на поверхности FC.

Увеличение или уменьшение показателя сопротивления раздиру в соответствии с добавлением наполнителя совпадает с изменениями показателя прочности резин.

Относительный гистерезис - важная эксплуатационная характеристика, характеризующая потери на качение, расход топлива и теплообразование. Определяли относительный гистерезис в соответствии с ГОСТ 252-75.

В таблице 2 приведены полученные значения относительного гистерезиса для резин с различными типами ЖОН в сравнении с эталонной. Из таблицы 2 видно, что значение относительного гистерезиса для эталонной резины составляет $11,2 \%$. Введение ЖОН снижает относительный гистерезис, за исключением FerroFlex, который в количестве больше 30 м. ч. начинает расти. Это можно объяснить в соответствии с представлениями об усилении, изложенными в статье [2], увеличением переходной зоны между частицей наполнителя и матрицей каучука.

Из рисунка 4 следует, что резины, худшие в статике, в усталости имеют существенно иное ранжирование. Что, например, для эталона говорит о маленьком разрывном удлинении. А для резины, содержащей наполнитель FF 40, напротив, о высоком значении удлинения при разрыве.
Из рисунка 6 видно, что резины, лучшие в статике, имеют аналогичное ранжирование и в области больших баз утомления. Это достаточно редкий случай, т. к. по статическим испытаниям нельзя сказать, как резины поведут себя в усталостных испытаниях.

Из рисунков 4-6 видно, что резины, которые имеют лучшие показали при статических деформациях, в усталостных испытаниях имеют другое ранжирование. Самая высокая усталостная выносливость у эталона.

По рисунку 4 можно увидеть, что резина «Эталон» и резина с малым количеством наполнителя FF 5 м.ч. имеют лучшие показатели и в статике, и в усталости.

Из рисунка 6 следует, что лучшей усталостной выносливостью обладают резины с наполнителями FC (0-100), 10 м.ч, FC (0-100), 20 м.ч.

Далее необходимо пересчитать кривые Веллера из режима заданных напряжений в режим заданных плотностей энергии деформации с помощью интегрирования кривых напряжениядеформации данных резин (рисунок 1). Из рисунка 6 (b) следует, что резины «эталон» и FC (0-20), 5 м.ч. имеют лучшие усталостные показатели при значении плотности энергии деформации равной 0,27 МПа. Однако при увеличении плотности энергии деформации до значения 0,32 МПа и более характер кривых меняется, и они располагаются ниже остальных, что свидетельствует о низкой усталостной выносливости. Из рисунков 4, 5, 6 видно, что ранжирование кривых FC (0-20) в разных режимах разное. В режиме заданных деформаций самая высокая усталостная выносливость у эталона и у резины FC (0-20), 5 м.ч. В режиме заданных напряжений самая высокая усталостная выносливость у резины с наполнителем FC (0-20), 20 м.ч., а вот у ранее упомянутых эталона и FC (0-20), 5 м.ч. - самая низкая ходимость.

Из рисунка 6 (c) следует, что резины с наполнителем FC (0-100) в статике и в динамике имеют существенно разное ранжирование. Из рисунков 4, 5, 6 можно увидеть, какая усталостная выносливость у резин с наполнителем FC (0-100) в разных режимах. В режиме заданных деформаций самая высокая усталостная выносливость у эталона и у резины FC (0-100), 10 м.ч. $\mathrm{B}$ режиме заданных напряжений самая высокая ходимость у резины FC (0-100), 20 м.ч. и FC (0-100), 10 м.ч. В режиме заданных плотностей энергии деформации при малой плотности энергии деформации самая высокая ходимость у эталона. Однако при увеличении плотности энергии деформации кривая снижается, что говорит о снижении усталостной выносливости. За исключением эталона в режиме заданных плотностей энергии деформации самая высокая ходимость у резин FC (0-100), 20 м.ч и FC (0-100), 10 м.ч. 
Из рисунка 7 (а) видно, что самая высокая усталостная выносливость при малых деформациях у эталона, самая низкая - у резины FF 5. Резины FF 5, FF 10, FF 20, FF 40 с учетом доверительного интервала имеют близкую друг к другу ходимость при данной (20,8\%) деформации.

По рисунку 7 (b) видно, самая высокая усталостная выносливость у резин «эталон» и FC (0-20) 5 м.ч. Самая низкая усталостная выносливость у резины FC (0-20) 24 м.ч. У резин FC (0-20) 10 м.ч и FC (0-20) 20 м.ч. близкая усталостная выносливость с учетом доверительного интервала.

Из рисунка 7 (с) видно, что в данном ряду самая высокая усталостная выносливость у эталона. Далее следуют FC (0-100) 10 м.ч, FC (0-100) 20 м.ч. и FC (0-100) 30, 5 м.ч., FC (0-100), 30 м.ч.

\section{Заключение}

Основные задачи построения усталостных кривых - оценка соответствия результатов прочностных испытаний (растяжение с постоянной скоростью до разрыва) усталостным по показателям усталостной долговечности, усталостной прочности, усталостной плотности энергии разрушения. Кроме того, стояла задача проверки возможности нанесения на одну кривую Веллера точек, полученных на разных приборах, - УР-500 и ЗПИ.

Основные выводы следующие.

- В результате проведённых исследований показано, что ранжирование резин в статике (растяжение с постоянной скоростью до разрушения) по всем трём показателям - разрывное удлинение, разрывное напряжение, разрывная плотность энергии деформации, вообще говоря, часто не совпадает друг с другом.

- Ранжирование резин в статике и в усталости также не совпадает друг с другом. Так как усталостные условия нагружения ближе к условиям реальной эксплуатации, чем статические, то для прогнозирования поведения РТИ в эксплуатации следует ориентироваться на результаты усталостных испытаний.

- Ранжирование резин по результатам усталостных испытаний в трёх разных режимах также часто не коррелирует между собой. Следовательно, прогнозирование ходимости резин следует проводить с учётом реального режима, ближе к которому эксплуатируется рассматриваемая деталь шины или РТИ.

- Совмещение результатов усталостных испытаний при многократном растяжении и при знакопеременном изгибе с вращением показало, что эта процедура правомерна, но только в тех случаях, когда температура саморазогрева на приборе ЗПИ не превышает некоторого критического значения, когда начинается термодеструкция.

- Результаты усталостных испытаний показали, что использование исследованных наполнителей при небольших степенях наполнения (до 30 м.ч.) может быть оправдано. В качестве примера можно привести Ferrocolor фракции 0-20 Мкм с массовой долей 5 м.ч. В этом случае усталостные свойства практически не ухудшаются по сравнению с промышленной резиной для массивных шин, в которую не добавлены новые наполнители.

\section{Литература}

1 Гамлицкий Ю.А. Наномеханика явления усиления наполненных эластомеров. // Каучук и резина. 2017 . № 5. C. 308-317.

2 Gamlitsky Yu.A. Nanomechanics of the Phenomenon of Reinforcement of Filled Elastomers // Nanomechanics Science and Technology An International Journal. 2013. V. 4. № 3. P. 1-18.

3 Черныш А.А., Яковлев С.Н. Экспериментальное исследование динамического модуля полиуретановых эластомеров при знакопеременном изгибе с вращением // Вестник Кузбасского государственного технического университета. 2018. № 4 (128).

4 Дружининская Ю.А. Усталостные свойства резин с использованием в их составе железооксидных и минеральных наполнителей: диссертация. Москва, 2019. 113 с.

5 Шашок Ж.С., Касперович А.В., Прокопчук Н.Р., Каюшников С.Н. Влияние рецептурного состава эластомерных композиций на упруго-деформационные свойства шинных резин // Труды БГТУ. 2013. № 4(160). С. 137-140.

6 Игуменова Т.И., Гудков М.А., Попов Г.В. Особенности усталостной устойчивости резин на основе комбинации минеральных наполнителей и фуллеренсодержащего технического углерода // Промышленное производство и использование эластомеров. 2012. № 1. С. 25-27.

7 Шульга А.М., Игуменова Т.И., Акатов Е.С. Влияние углеродных наноматериалов на усталостные свойства полимерных композиций // Известия вузов. Прикладная химия и биотехнология. 2016. № 4 (19). С. 169-172.

8 Dong B., Zhang L., Wu Y. Influences of different dimensional carbon-based nanofillers on fracture and fatigue resistance of natural rubber composites // Polymer Testing. 2017. V. 63. P. 281-288.

9 Tonatto M.L.P., Forte M.M.C., Amico S.C. Compressive-tensile fatigue behavior of cords/rubber composites // Polymer Testing. 2017. V. 61. P. 185-190.

10 Marco Y. et al. Prediction of fatigue properties of natural rubber based on the descriptions of the cracks population and of the dissipated energy // Polymer Testing. 2017. V. 59. P. 67-74. 


\section{References}

1 Gamlitsky Yu.A. Nanomechanics of the phenomenon of reinforcement of filled elastomers. Rubber and Rubber. 2017. no. 5. pp. 308-317. (in Russian).

2 Gamlitsky Yu.A. Nanomechanics of the Phenomenon of Reinforcement of Filled Elastomers. Nanomechanics Science and Technology. An International Journal. 2013. vol. 4. no. 3. pp. 1-18.

3 Chernysh A.A., Yakovlev S.N. An experimental study of the dynamic modulus of polyurethane elastomers under alternating bending with rotation. Bulletin of the Kuzbass State Technical University. 2018. no. 4 (128). (in Russian).

4 Druzhininskaya Yu.A. Fatigue properties of rubbers using iron oxide and mineral fillers in their composition: dissertation. Moscow, 2019. 113 p. (in Russian).

5 Shashok J.S., Kasperovich A.V., Prokopchuk N.R., Kaushnikov S.N. The influence of the prescription composition of elastomeric compositions on the elastic-deformation properties of tire rubbers. Transactions of BSTU. 2013. no. 4 (160). pp. 137-140. (in Russian).

6 Igumenova T.I., Gudkov M.A., Popov G.V. Features of the fatigue resistance of rubbers based on a combination of mineral fillers and fullerene-containing carbon black. Industrial production and use of elastomers. 2012. no. 1. pp. 25-27. (in Russian).

7 Shulga A.M., Igumenova T.I., Akatov E.S. Influence of carbon nanomaterials on the fatigue properties of polymer compositions. News of Universities. Applied Chemistry and Biotechnology. 2016. no. 4 (19). pp. 169-172. (in Russian).

8 Dong B., Zhang L., Wu Y. Influences of different dimensional carbon-based nanofillers on fracture and fatigue resistance of natural rubber composites. Polymer Testing. 2017. vol. 63. pp. 281-288.

9 Tonatto M.L.P., Forte M.M.C., Amico S.C. Compressive-tensile fatigue behavior of cords/rubber composites. Polymer Testing. 2017. vol. 61. pp. 185-190.

10 Marco Y. et al. Prediction of fatigue properties of natural rubber based on the descriptions of the cracks population and of the dissipated energy. Polymer Testing. 2017. vol. 59. pp. 67-74.

\section{Сведения об авторах}

Инна А. Литвинова аспирант, кафедра химии и технологии переработки эластомеров имени Ф.Ф. Кошелева, МИРЭА Российский технологический университет, пр-т Вернадского, д. 78, г. Москва, 119454, Россия, innalitv@ mail.ru (Dhttps://orcid.org/0000-0001-5359-1631

Игорь В. Веселов к.т.н., профессор, ООО НПКЦ ВЕСКОМ, ул. Буракова, 27, г. Москва, 105118, Россия, iv-veselov@mail.ru Юрий А. Гамлицкий к.ф-м.н., доцент, кафедра химии и технологии переработки эластомеров имени Ф.Ф. Кошелева, ООО НПКЦ ВЕСКОМ, ул. Буракова, 27, г. Москва, 105118, Россия, gamlit48@mail.ru

Dhttps://orcid.org/0000-0001-8925-2439

\section{Вклад авторов}

Инна А. Литвинова выполнила расчёты, провела эксперимент, обзор литературы по исследуемой проблеме

Игорь В. Веселов написал рукопись, корректировал её до подачи в редакцию и несёт ответственность за плагиат

Юрий А. Гамлицкий консультация в ходе исследования Конфликт интересов

Авторы заявляют об отсутствии конфликта интересов.

\section{Information about authors}

Inna A. Litvinova graduate student, chemistry and technology of processing elastomers named after F.F. Kosheleva department, MIREA - Russian Technological University, 78 Vernadskogo Ave., Moscow, 119454, Russia, innalitv@mail.ru (Dhttps://orcid.org/0000-0001-5359-1631

Igor V. Veselov Cand. Sci. (Engin.), professor, NPKTS VESKOM LLC, ul. Burakova, 27, Moscow, 105118, Russia, iv-veselov@mail.ru

Yuriy A. Gamlitskiy Cand. Sci. (Phys.-Math.), associate professor, chemistry and technology of processing elastomers named after F.F. Kosheleva department, NPKTS VESKOM LLC, ul. Burakova, 27, Moscow, 105118, Russia, gamlit48@mail.ru Dhttps://orcid.org/0000-0001-8925-2439

\section{Contribution}

Inna A. Litvinova review of the literature on an investigated problem, conducted an experiment, performed computations

Igor V. Veselov wrote the manuscript, correct it before filing in editing and is responsible for plagiarism

Yuriy A. Gamlitskiy consultation during the study

Conflict of interest

The authors declare no conflict of interest.

\begin{tabular}{ccc}
\hline Поступила 04/10/2019 & После редакции 14/11/2019 & Принята в печать 25/11/2019 \\
\hline Received 04/10/2019 & Accepted in revised 14/11/2019 & Accepted 25/11/2019 \\
\hline
\end{tabular}

